\title{
Significance of aneurysm wall enhancement on high- -resolution vessel wall magnetic resonance imaging in clinical management of patients with intracranial aneurysms
}

\author{
Łukasz Zwarzany, Marcin Sawicki, Wojciech Poncyljusz \\ Department of Diagnostic Imaging and Interventional Radiology, Pomeranian Medical University, Szczecin, Poland
}

\begin{abstract}
Introduction. The prevalence of intracranial aneurysms is estimated to be around $3 \%$ in the general population. Although these are often incidental findings, they potentially carry the risk of rupture, with all of the devastating consequences of SAH.

State of the art. Several risk factors of aneurysm rupture have been identified, including aneurysm size, irregular shape, and location. Although it is widely accepted that the risk of rupture increases with size, small aneurysms remain the cause of a significant percentage of aneurysmal SAH. Up to $30 \%$ of patients with acute aneurysmal SAH have multiple aneurysms. Determining the site of rupture in these patients can be challenging, given that the results of imaging studies and clinical symptoms are sometimes inconclusive. It would be extremely useful to identify new imaging biomarkers of aneurysm instability which could have an impact on patient management and qualification for treatment. High-resolution vessel wall magnetic resonance imaging (HR-VW MRI) opens up new possibilities for improved characterisation of intracranial vasculature. One of the most promising clinical applications of this new imaging tool is the evaluation of intracranial aneurysms.

Clinical implications. Aneurysm wall enhancement (AWE) on HR-VW MRI is believed to be a marker for wall inflammation and, potentially, for aneurysm instability. In this article, we summarise the published literature on AWE with special emphasis on its use in determining the site of rupture in the setting of acute $\mathrm{SAH}$ in patients with multiple aneurysms, as well as its role in identifying unruptured aneurysms which are at the greatest risk of rupture.

Future directions. More and larger studies are needed to definitively establish the role of AWE on HR-VW MRI in the diagnostic workup of patients with intracranial aneurysms.
\end{abstract}

Key words: intracranial aneurysm, aneurysm wall enhancement, magnetic resonance imaging, high-resolution vessel wall magnetic resonance imaging

(Neurol Neurochir Pol 2020; 54 (6): 518-523)

\section{Introduction}

The prevalence of intracranial aneurysms is estimated to be around $3 \%$ of the general population [1]. With the number of imaging studies, and their availability, constantly increasing, so the number of newly diagnosed cases grows. Although these are often incidental findings, they potentially carry the risk of rupture, with all of the devastating consequences of SAH. Several risk factors of aneurysm rupture have been identified, including aneurysm size, irregular shape, and location $[2,3]$.
It is generally accepted that the risk of rupture increases with size. However, small aneurysms remain the cause of a significant percentage of aneurysmal SAH [4]. Up to $30 \%$ of patients with acute aneurysmal SAH have multiple intracranial aneurysms $[5,6]$. Correct identification of a ruptured aneurysm in this condition is crucial for treatment planning. The diagnosis is mainly based on clinical symptoms plus noninvasive imaging studies such as CT and CTA $[7,8]$. However, determining the site of rupture in these patients is sometimes challenging [9]. It would be extremely useful to identify new imaging biomarkers

Address for correspondence: Łukasz Zwarzany, Department of Diagnostic Imaging and Interventional Radiology, Pomeranian Medical University, Unii Lubelskiej 1 Str., 71-252 Szczecin, Poland, e-mail: zwarzany@gmail.com 


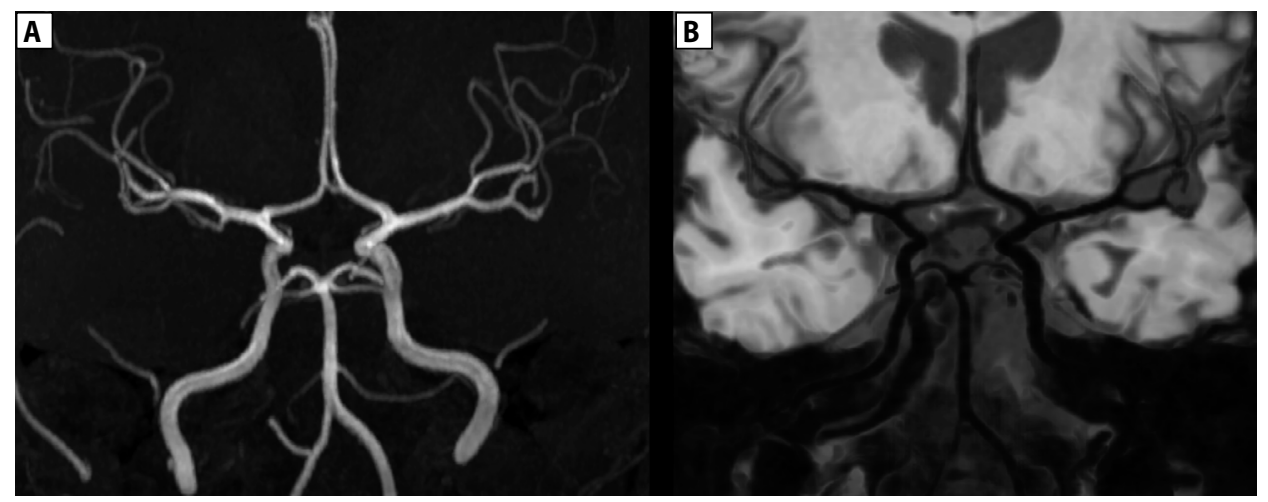

Figure 1. A. Time-of-flight (TOF) MR angiography (MRA) of a healthy volunteer. B. Corresponding minimum intensity projection (MinIP) reconstruction of precontrast HR-VW MRI demonstrating circle of Willis. Source: Department of Diagnostic Imaging and Interventional Radiology, Pomeranian Medical University

of aneurysm instability which could have an impact on patient management and qualification for treatment.

High-resolution vessel wall magnetic resonance imaging (HR-VW MRI) opens up new possibilities for improved characterisation of intracranial vasculature [10]. It complements the vascular imaging techniques available thus far, and allows us to see beyond the vessel lumen. Vessel wall thickening and enhancement represent the key imaging findings that can be identified by radiologists on HR-VW MRI. This novel imaging tool is based on the acquisition of high spatial resolution images with flowing blood signal suppression. The acquisition of isotropic (0.4-0.7 mm voxel size range) $3 \mathrm{D}$ sequences, with subsequent multiplanar reconstructions, is the most commonly used approach. Due to the technical demands of HR-VW MRI, a high-field (at least 3T) MRI scanner is necessary for optimal image quality. An MRI protocol consisting of time-of-flight angiography, high-resolution 3D pre- and postcontrast T1-weighted fast spin-echo images covering the circle of Willis and its proximal branches, can be performed in less than 30 minutes (Fig. 1).

With the ever-increasing availability of high-field MRI scanners, HR-VW MRI is moving from research laboratories into clinical practice. One of the most promising clinical applications of this imaging tool is the evaluation of intracranial aneurysms. In this review, we present the results of published studies regarding the use of HR-VW MRI for the evaluation of both ruptured and unruptured aneurysms.

\section{State of the art}

Matouk et al. first described their experience with HRVW MRI in the setting of acute aneurysmal SAH [11]. The authors hypothesised that a ruptured aneurysm might show wall enhancement on HR-VW MRI. Aneurysm wall enhancement (AWE) was found in all five investigated cases. This small case series also demonstrated the potential of HR-VWI MRI in determining the site of rupture in patients harbouring multiple aneurysms. In three such patients, wall enhancement was limited to the ruptured aneurysm only. The authors suggested, based on the results of previous histological analysis of ruptured and unruptured aneurysms, that an inflammatory process, along with physical disruption of the vessel wall, might be responsible for AWE.

Larger studies have shown that wall enhancement is a frequent imaging feature of ruptured aneurysms [12-17]. Researchers from Japan detected strong and faint wall enhancement in $73.8 \%$ and $24.6 \%$ of 61 ruptured aneurysms, respectively [12]. Of 61 patients with SAH included in this study, 11 were diagnosed with multiple aneurysms: in all of them, the site of rupture was confirmed intraoperatively. Interestingly, in 15 patients wall enhancement was limited to the bleed or apex of the ruptured aneurysm. Overall, the sensitivity and specificity of AWE for the detection of a ruptured aneurysm was $98.4 \%$ and $81.9 \%$, respectively.

Data from the literature shows that HR-VW MRI can indicate the most probable rupture site in patients with both multiple aneurysms and SAH in situations when other imaging findings (e.g. pattern of SAH, aneurysm size, morphology) are inconclusive. Kondo et al. published a case report of a 68-year-old woman who presented with SAH and was diagnosed with two intracranial aneurysms [18]. HR-VW MRI showed strong wall enhancement of a smaller anterior communicating artery aneurysm and no wall enhancement of a larger basilar tip aneurysm, which was initially suspected to be the rupture site. It was confirmed intraoperatively that the anterior communicating artery aneurysm was the source of intracranial haemorrhage.

In most of the published series, AWE has been analysed in a qualitative manner using different grading scales $[12,15$, 17]. However, quantitative methods have also been developed $[13,14]$. Omodaka et al. used the wall enhancement index and the contrast ratio of the circumference aneurysm wall against the stalk to characterise the imaging findings of $26 \mathrm{SAH}$ patients with multiple intracranial aneurysms [13]. The authors 
demonstrated that stronger AWE on HR-VW MRI is associated with aneurysm ruptured status, independent of aneurysm size and patient characteristics. The developed quantitative methods achieved sensitivity and specificity rates of $100 \%$ and $69 \%$, respectively, for the detection of a ruptured aneurysm. It is worth noting that in approximately one in four patients, the ruptured aneurysm was not the largest one.

HR-VW MRI has been also investigated in the setting of SAH with no identifiable haemorrhagic source. Coutinho et al. performed HR-VW MRI in 11 patients with an angiogram-negative non-perimesencephalic SAH [19]. Although it did not alter the management of the patients, HR-VW MRI revealed abnormalities in seven cases. They were characterised as focal and contiguous with the outer margin of the basilar artery walls in two patients. The authors argued that these imaging findings could have represented a thrombosed saccular aneurysm or a ruptured blood blister aneurysm.

Research is ongoing to prove that wall enhancement on HR-VW MRI is associated with aneurysm instability, and the results of already published papers are promising. In one of the earliest studies in this field, Edjlali et al. investigated 108 aneurysms with HR-VW MRI [15]. The aneurysms were categorised as either stable (incidental and nonevolving) or unstable (ruptured, symptomatic, or undergoing morphological modification). Wall enhancement was significantly more frequently observed in unstable than in stable aneurysms, in $87 \%$ and $28.5 \%$ of cases, respectively. Furthermore, statistical analysis showed that AWE was the only independent feature to be related to unstable status. Interestingly, no association was found between AWE and aneurysm size. This might have been the result of the heterogeneity of the studied population, which included both ruptured and unruptured aneurysms.

In contrast to Edjlali et al. [15], the correlation between AWE and aneurysm size has been frequently reported in other papers [20-23]. Liu et. al, who analysed 61 unruptured aneurysms, found wall enhancement in all of the aneurysms larger than $13 \mathrm{~mm}$, but in only $12 \%$ of the aneurysms smaller than $7 \mathrm{~mm}$ [20]. According to Backes et al., aneurysm size is the strongest determinant of wall enhancement in unruptured aneurysms [21]. Fu et al. showed that AWE is more common in symptomatic patients presenting with sentinel headaches and third nerve palsy [24]. Generally, data from the literature demonstrates the relationship between AWE and the established risk factors for aneurysm rupture. The PHASES (Population, Hypertension, Age, Size, Earlier SAH, Site) score is a tool developed to predict the 5-year risk of aneurysm rupture [25]. More than one paper has reported this risk to be significantly higher in aneurysms with wall enhancement compared to aneurysms without it $[22,23]$.

Several other studies have compared ruptured to unruptured aneurysms in terms of wall enhancement on HR-VW MRI [12, 14-17]. Omodaka et al. noted that although AWE is a sensitive radiological marker of ruptured aneurysms, its specificity is quite low, as it is also found in a significant number of unruptured aneurysms [16]. Therefore, they used quantitative measures to analyse wall enhancement of 104 aneurysms (28 ruptured, 76 unruptured) and showed that it is significantly higher in ruptured than in unruptured aneurysms.

Edjlali et al., in their more recent study including 333 aneurysms (26 ruptured, 307 unruptured), also decided to evaluate the degree of AWE [17]. They used a four-grade classification of AWE and showed that a thick circumferential pattern has the highest specificity for differentiating between stable and unstable unruptured aneurysms. Overall, wall enhancement was detected in $88.5 \%$ of ruptured, in $71 \%$ of unstable unruptured, and in $38.4 \%$ of stable unruptured aneurysms.

Recently, Vergouwen et al. published results of the first longitudinal study investigating AWE, as opposed to previous studies in this field which were all cross-sectional in design [26]. Fifty-seven patients with 65 unruptured aneurysms were followed-up for a median of 27 months. Of 19 aneurysms showing wall enhancement at baseline HR-VW MRI, two aneurysms ruptured, and two aneurysms demonstrated growth, during the course of the study. All aneurysms without wall enhancement were stable at follow-up angiography. This is to date the only study to have directly shown that AWE on HR-VW MRI may indicate a higher risk for aneurysm rupture.

Unfortunately, only a few studies including a limited number of patients with intracranial aneurysms have correlated HR-VW MRI and histopathological findings [27-32]. In the study by Larsen et al., aneurysms with strong wall enhancement on HR-VW MRI exhibited inflammatory cell infiltration, neovascularisation and the presence of vasa vasorum [29]. None of these histopathological findings were present in aneurysms without wall enhancement. In a more recent study of 54 unruptured intracranial aneurysms, the presence of AWE was also associated with inflammatory infiltration of the aneurysm wall [30]. The authors stated that a focal, rather than uniform, pattern of AWE may indicate atherosclerotic plaque formation. On the other hand, Matsushige et al. investigated ruptured aneurysms and suggested that focal AWE may represent a fresh intraluminal thrombus, whereas circumferential AWE may indicate neovascularisation and infiltration of inflammatory cells in the aneurysm wall [32].

\section{Clinical implications}

Aneurysm wall enhancement is a common imaging finding on HR-VW MRI in patients with ruptured intracranial aneurysms [12-17]. In patients with SAH and multiple aneurysms, AWE on HR-VW MRI can identify the rupture site with high sensitivity but low specificity $[12,13]$. This low specificity is due to the fact that AWE is also observed in a significant number of unruptured aneurysms. Thus, caution must be taken when using this new imaging tool to determine the site of rupture in patients with SAH and multiple aneurysms. Moreover, based on our experience, often more than one aneurysm shows wall enhancement on HR-VW MRI in patients with 

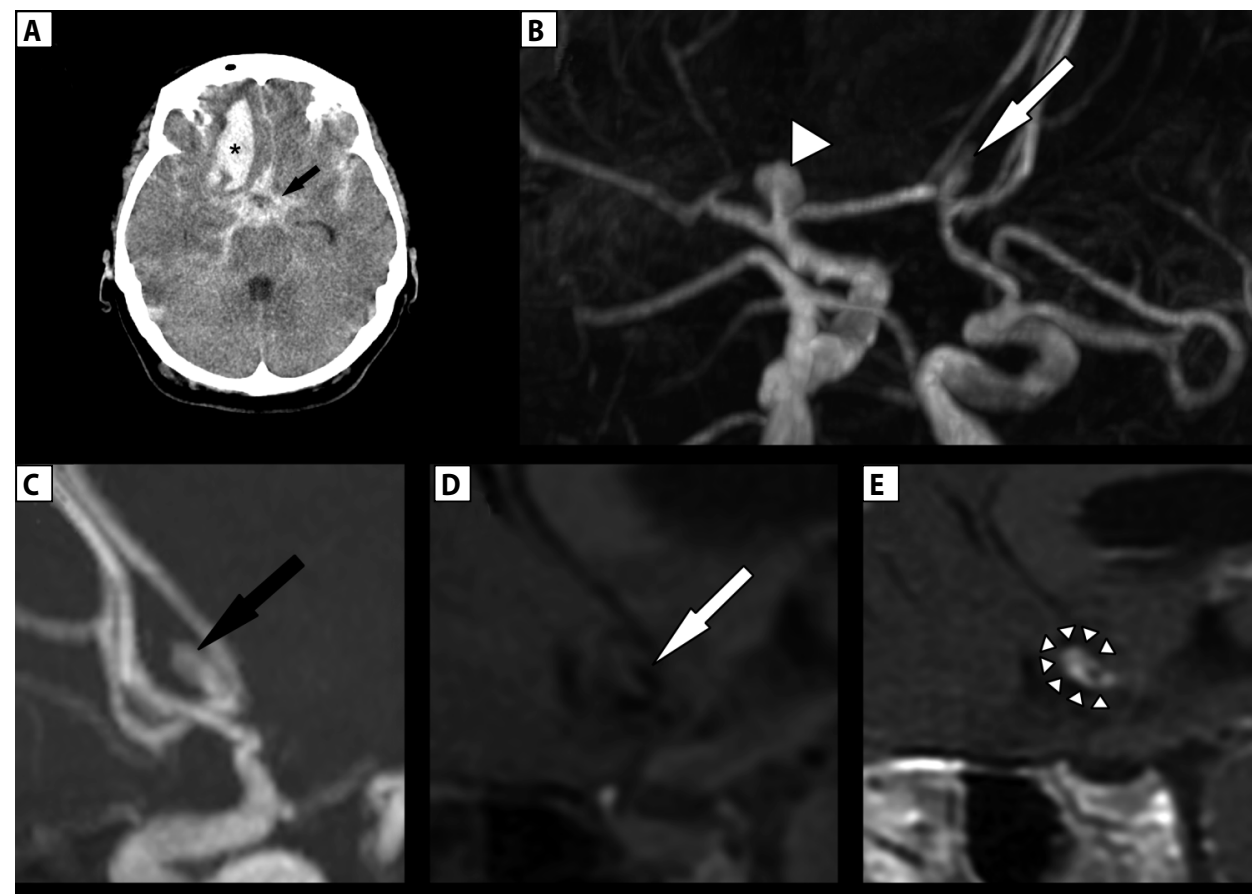

D

E

$\mathbf{F}$

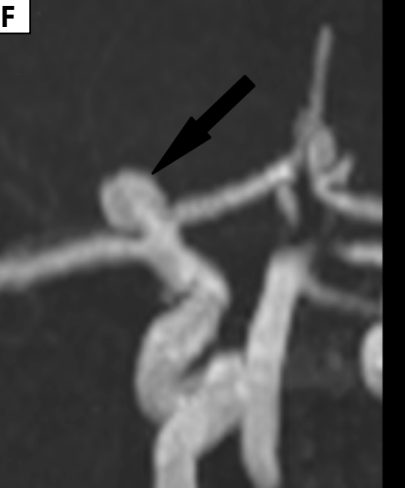

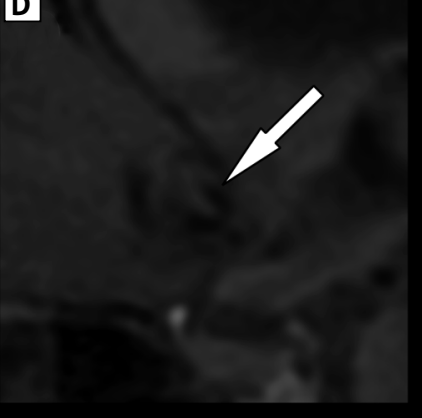

G

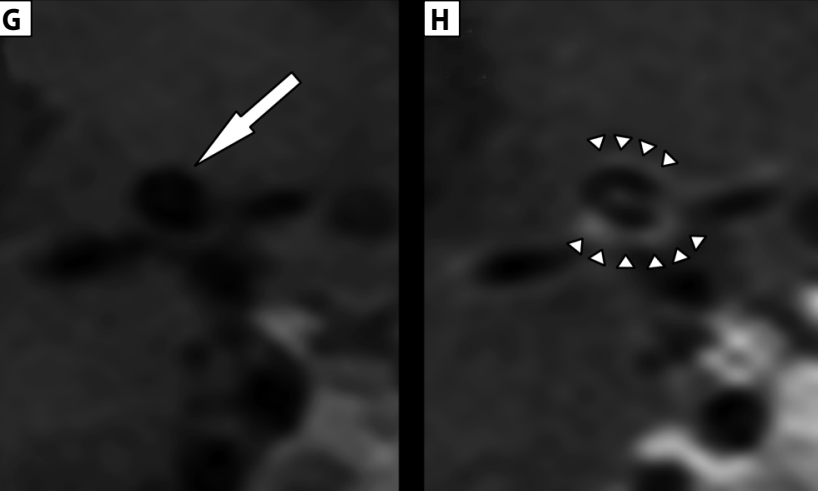

Figure 2.54-year-old woman with severe headache of sudden onset. A. Noncontrast head CT reveals diffuse subarachnoid haemorrhage (arrow) with intraparenchymal haemorrhage in right frontal lobe (asterisk). B. 3D reconstruction of time-of-flight (TOF) MR angiography (MRA) shows two aneurysms arising at ACOA (arrow) and at bifurcation of right ICA (arrowhead). C, D. TOF MRA and precontrast HR-VW MRI demonstrating ACoA aneurysm (arrow). E. ACoA aneurysm shows thick wall enhancement on postcontrast HR-VW MRI (arrowheads). F, G. TOF MRA and precontrast HR-VW MRI demonstrating right ICA aneurysm (arrow). H. Right ICA aneurysm also shows wall enhancement on postcontrast HR-VW MRI (arrowheads), but to a lesser degree than ACoA aneurysm. Although ACoA aneurysm was deemed to be most likely site of rupture, both aneurysms were occluded during endovascular coiling (not shown) as they both showed wall enhancement on HR-VW MRI. Source: Department of Diagnostic Imaging and Interventional Radiology, Pomeranian Medical University

multiple aneurysms, something which further complicates the therapeutic decision-making process (Fig. 2).

However, the most promising clinical application of HRVW MRI in patients with intracranial aneurysms is the identification of aneurysms which are at greater risk of rupture. In our view, radiologists and referring clinicians should be aware of several issues before incorporating this imaging tool into daily clinical practice $[33,34]$. Firstly, we do not really know if the enhancement we see on HR-VW MRI, either circumferential or focal, at the aneurysm periphery is indeed within its wall. According to histological analysis of intracranial aneurysms, their wall may be as thin as $0.02 \mathrm{~mm} \mathrm{[35],} \mathrm{which} \mathrm{is}$ far beyond the spatial resolution of images obtained with 3T, or even 7T, MRI systems. Furthermore, it was demonstrated recently in a phantom-based MRI study that a slow flow which is insufficiently suppressed with currently available techniques, can mimic AWE on postcontrast HR-VW MRI images [36]. This finding may hold true for in vivo conditions because large and irregular aneurysms, which are more likely to present with slow and turbulent flow, show wall enhancement on HR-VW MRI more frequently [20-23].

In our practice, we have encountered several cases of aneurysms which were not suitable for coiling and were treated with stent implantation only and showed strong 
wall enhancement on short-term follow-up HR-VW MRI. It cannot be excluded that this enhancement might have been the result of the flow-diversion effect of the implanted device (Supplementary Fig. 1).

Although the results and conclusions of published studies are quite encouraging, they need robust critical evaluation. In some observational studies, a misleading assumption has been made that AWE is an imaging biomarker of aneurysm instability in general, based mainly on its high prevalence in ruptured aneurysms $[15,17]$. Without prior imaging studies, one cannot determine whether or not AWE was present before the aneurysm rupture. In ruptured aneurysms, it might simply be a sequel of vessel wall disruption. Furthermore, demonstrating the association of AWE on HR-VW MRI with larger aneurysm size or irregular aneurysm shape has no practical clinical value because these features are already themselves well-documented risk factors of aneurysm rupture [20-23].

To the best of our knowledge, there is only one prospective study to have evaluated the natural history of aneurysms with wall enhancement on HR-VW MRI [26]. However, a significant limitation of this study was a relatively small sample size. Finally, heterogeneity in the design of studies, including differences in acquisition protocols and AWE scoring systems, makes comparisons difficult.

\section{Future directions}

Multi-centre, prospective studies with a standardised imaging protocol are needed to definitely establish the clinical role of HR-VW MRI in the diagnostic workup of patients with intracranial aneurysms. Comparing the natural history of aneurysms with and without wall enhancement on HR-VW MRI will probably answer the question of whether the presence of this imaging sign is indeed an indicator of aneurysm instability.

Furthermore, to better understand this imaging finding, correlation with histological analysis will be essential in future studies. This, however, may be difficult to achieve because a significant number of aneurysms are treated with the endovascular approach. An investigation of aneurysm flow dynamics and its impact on AWE is also crucial. Further development of slow flow suppression techniques, such as preparation pulses, would be undoubtedly beneficial.

Ethical permission: Ethical approval was not necessary for the preparation of this review article.

Funding: This publication was prepared without any external source of funding.

Conflicts of interest: The authors declare that there is no conflict of interest.

\section{References}

1. Vlak MHm, Algra A, Brandenburg R, et al. Prevalence of unruptured intracranial aneurysms, with emphasis on sex, age, comorbidity, country, and time period: a systematic review and meta-analysis. Lancet
Neurol. 2011; 10(7): 626-636, doi: 10.1016/S1474-4422(11)70109-

0, indexed in Pubmed: 21641282.

2. Wiebers DO, Whisnant JP, Huston J, et al. International Study of Unruptured Intracranial Aneurysms Investigators. Unruptured intracranial aneurysms: natural history, clinical outcome, and risks of surgical and endovascular treatment. Lancet. 2003; 362(9378): 103-110, doi: 10.1016/s0140-6736(03)13860-3, indexed in Pubmed: 12867109.

3. Backes D, Vergouwen MDI, Velthuis BK, et al. Difference in aneurysm characteristics between ruptured and unruptured aneurysms in patients with multiple intracranial aneurysms. Stroke. 2014; 45(5): 1299-1303, doi: 10.1161/STROKEAHA.113.004421, indexed in Pubmed: 24652309.

4. Bender MT, Wendt H, Monarch T, et al. Small Aneurysms Account for the Majority and Increasing Percentage of Aneurysmal Subarachnoid Hemorrhage: A 25-Year, Single Institution Study. Neurosurgery. 2018; 83(4): 692-699, doi: 10.1093/neuros/nyx484, indexed in Pubmed: 29029314.

5. Juvela S. Risk factors for multiple intracranial aneurysms. Stroke. 2000; 31(2): 392-397, doi: 10.1161/01.str.31.2.392, indexed in Pubmed: 10657411.

6. Sawicki M, Kościukiewicz K, Jeżewski D, et al. Diagnostic value of non-enhanced computed tomography in identifying location of ruptured cerebral aneurysm in patients with aneurysmal subarachnoid haemorrhage. Neurol Neurochir Pol. 2020; 54(1): 47-53, doi: 10.5603/ PJNNS.a2020.0007, indexed in Pubmed: 31967317.

7. WOOD EH. ANGIOGRAPHIC IDENTIFICATION OF THE RUPTURED LESION IN PATIENTS WITH MULTIPLE CEREBRAL ANEURYSMS. J Neurosurg. 1964; 21: 182-198, doi: 10.3171/jns.1964.21.3.0182, indexed in Pubmed: 14127619.

8. Nehls DG, Flom RA, Carter LP, et al. Multiple intracranial aneurysms: determining the site of rupture. J Neurosurg. 1985; 63(3): 342-348, doi: 10.3171/jns.1985.63.3.0342, indexed in Pubmed: 4020459.

9. Hino $\mathrm{A}$, Fujimoto $\mathrm{M}$, Iwamoto $\mathrm{Y}$, et al. False localization of rupture site in patients with multiple cerebral aneurysms and subarachnoid hemorrhage. Neurosurgery. 2000; 46(4): 825-830, doi: 10.1097/00006123-200004000-00011, indexed in Pubmed: 10764255.

10. Mandell DM, Mossa-Basha M, Qiao Y, et al. Vessel Wall Imaging Study Group of the American Society of Neuroradiology. Intracranial Vessel Wall MRI: Principles and Expert Consensus Recommendations of the American Society of Neuroradiology. AJNR Am J Neuroradiol. 2017; 38(2): 218-229, doi: 10.3174/ajnr.A4893, indexed in Pubmed: 27469212.

11. Matouk CC, Mandell DM, Günel M, et al. Vessel wall magnetic resonance imaging identifies the site of rupture in patients with multiple intracranial aneurysms: proof of principle. Neurosurgery. 2013; 72(3): 492-6; discussion 496, doi: 10.1227/NEU.0b013e31827d1012, indexed in Pubmed: 23151622.

12. Nagahata S, Nagahata M, Obara M, et al. Wall Enhancement of the Intracranial Aneurysms Revealed by Magnetic Resonance Vessel Wall Imaging Using Three-Dimensional Turbo Spin-Echo Sequence with Motion-Sensitized Driven-Equilibrium: A Sign of Ruptured Aneurysm? Clin Neuroradiol. 2016; 26(3): 277-283, doi: 10.1007/s00062-0140353-z, indexed in Pubmed: 25332151.

13. Omodaka S, Endo H, Niizuma K, et al. Circumferential Wall Enhancement on Magnetic Resonance Imaging is Useful to Identify Rupture Site in Patients with Multiple Cerebral Aneurysms. Neurosurgery. 2018; 82(5): 638-644, doi: 10.1093/neuros/nyx267, indexed in Pubmed: 28586440. 
14. Wang GX, Wen Li, Lei S, et al. Wall enhancement ratio and partial wall enhancement on MRI associated with the rupture of intracranial aneurysms. J Neurointerv Surg. 2018; 10(6): 566-570, doi: 10.1136/ neurintsurg-2017-013308, indexed in Pubmed: 28918385.

15. Edjlali M, Gentric JC, Régent-Rodriguez C, et al. Does aneurysmal wall enhancement on vessel wall MRI help to distinguish stable from unstable intracranial aneurysms? Stroke. 2014; 45(12): 37043706, doi: 10.1161/STROKEAHA.114.006626, indexed in Pubmed: 25325912.

16. Omodaka S, Endo H, Niizuma K, et al. Quantitative Assessment of Circumferential Enhancement along the Wall of Cerebral Aneurysms Using MR Imaging. AJNR Am J Neuroradiol. 2016; 37(7): 1262-1266, doi: 10.3174/ajnr.A4722, indexed in Pubmed: 26939634.

17. Edjlali M, Guédon A, Ben Hassen W, et al. Circumferential Thick Enhancement at Vessel Wall MRI Has High Specificity for Intracranial Aneurysm Instability. Radiology. 2018; 289(1): 181-187, doi: 10.1148/ radiol.2018172879, indexed in Pubmed: 29969070.

18. Kondo R, Yamaki T, Mouri W, et al. [Magnetic resonance vessel wall imaging reveals rupture site in subarachnoid hemorrhage with multiple cerebral aneurysms]. No Shinkei Geka. 2014; 42(12): 1147-1150, doi: 10.11477/mf.1436200050, indexed in Pubmed: 25433063.

19. Coutinho JM, Sacho RH, Schaafsma JD, et al. High-Resolution Vessel Wall Magnetic Resonance Imaging in Angiogram-Negative Non-Perimesencephalic Subarachnoid Hemorrhage. Clin Neuroradiol. 2017; 27(2): 175-183, doi: 10.1007/s00062-015-0484-x, indexed in Pubmed: 26608742.

20. Liu P, Qi H, Liu A, et al. Relationship between aneurysm wall enhancement and conventional risk factors in patients with unruptured intracranial aneurysms: A black-blood MRI study. Interv Neuroradiol. 2016; 22(5): 501-505, doi: 10.1177/1591019916653252, indexed in Pubmed: 27341856.

21. Backes D, Hendrikse J, van der Schaaf I, et al. Determinants of Gadolinium-Enhancement of the Aneurysm Wall in Unruptured Intracranial Aneurysms. Neurosurgery. 2018; 83(4): 719-725, doi: 10.1093/ neuros/nyx487, indexed in Pubmed: 29088454.

22. Lv N, Karmonik C, Huang $Q$, et al. Relationship Between Aneurysm Wall Enhancement in Vessel Wall Magnetic Resonance Imaging and Rupture Risk of Unruptured Intracranial Aneurysms. Neurosurgery. 2019; 84(6): E385-E391, doi: 10.1093/neuros/nyy310, indexed in Pubmed: 30011026.

23. Hartman JB, Watase H, Sun J, et al. Intracranial aneurysms at higher clinical risk for rupture demonstrate increased wall enhancement and thinning on multicontrast 3D vessel wall MRI. Br J Radiol. 2019; 92(1096): 20180950, doi: 10.1259/bjr.20180950, indexed in Pubmed: 30653339.

24. Fu Q, Guan S, Liu C, et al. Clinical Significance of Circumferential Aneurysmal Wall Enhancement in Symptomatic Patients with Unruptured Intracranial Aneurysms: a High-resolution MRI Study. Clin Neuroradiol. 2018; 28(4): 509-514, doi: 10.1007/s00062-017-0598-4, indexed in Pubmed: 28656370.
25. Greving J, Wermer M, Brown R, et al. Development of the PHASES score for prediction of risk of rupture of intracranial aneurysms: a pooled analysis of six prospective cohort studies. The Lancet Neurology. 2014; 13(1): 59-66, doi: 10.1016/s1474-4422(13)70263-1.

26. Vergouwen MDI, Backes D, van der Schaaf IC, et al. Gadolinium Enhancement of the Aneurysm Wall in Unruptured Intracranial Aneurysms Is Associated with an Increased Risk of Aneurysm Instability: A Follow-Up Study. AJNR Am J Neuroradiol. 2019; 40(7): 1112-1116, doi: 10.3174/ajnr.A6105, indexed in Pubmed: 31221634.

27. Hu P, Yang Qi, Wang DD, et al. Wall enhancement on high-resolution magnetic resonance imaging may predict an unsteady state of an intracranial saccular aneurysm. Neuroradiology. 2016; 58(10): 979-985, doi: 10.1007/s00234-016-1729-3, indexed in Pubmed: 27438805.

28. Shimonaga K, Ishii D, Matsushige T, et al. Clinicopathological Insights From Vessel Wall Imaging of Unruptured Intracranial Aneurysms. Stroke. 2018; 49(10): 2516-2519, doi: 10.1161/STROKEAHA.118.021819, indexed in Pubmed: 30355091.

29. Larsen N, von der Brelie C, Trick D, et al. Vessel Wall Enhancement in Unruptured Intracranial Aneurysms: An Indicator for Higher Risk of Rupture? High-Resolution MR Imaging and Correlated Histologic Findings. AJNR Am J Neuroradiol. 2018; 39(9): 1617-1621, doi: 10.3174/ajnr.A5731, indexed in Pubmed: 30026386.

30. Quan K, Song J, Zhu W, et al. Validation of Wall Enhancement as a New Imaging Biomarker of Unruptured Cerebral Aneurysm. Stroke. 2019; 50(6): 1570-1573, doi: 10.1161/STROKEAHA.118.024195, indexed in Pubmed: 31035900.

31. Hudson JS, Zanaty M, Nakagawa D, et al. Magnetic Resonance Vessel Wall Imaging in Human Intracranial Aneurysms. Stroke. 2018 [Epub ahead of print]: STROKEAHA118023701, doi: 10.1161/STROKEAHA.118.023701, indexed in Pubmed: 30580739.

32. Matsushige T, Shimonaga K, Mizoue T, et al. Focal Aneurysm Wall Enhancement on Magnetic Resonance Imaging Indicates Intraluminal Thrombus and the Rupture Point. World Neurosurg. 2019; 127: e578-e584, doi: 10.1016/j.wneu.2019.03.209, indexed in Pubmed: 30928597.

33. Lehman VT, Brinjikji W. Vessel Wall Imaging of Unruptured Intracranial Aneurysms: Ready for Prime Time? Not so Fast! AJNR Am J Neuroradiol. 2019; 40(6): E26-E29, doi: 10.3174/ajnr.A6048, indexed in Pubmed: 31048296.

34. van den Berg R, Cornelissen BMW, Leemans EL, et al. Vessel wall enhancement of intracranial aneurysms: fact or artifact? Neurosurg Focus. 2019; 47(1): E18-270, doi: 10.3171/2019.4.FOCUS19236, indexed in Pubmed: 31261122.

35. Suzuki J, Ohara H. Clinicopathological study of cerebral aneurysms. Origin, rupture, repair, and growth. J Neurosurg. 1978; 48(4): 505514, doi: 10.3171/jns.1978.48.4.0505, indexed in Pubmed: 632875.

36. Cornelissen BMW, Leemans EL, Coolen BF, et al. Insufficient slow-flow suppression mimicking aneurysm wall enhancement in magnetic resonance vessel wall imaging: a phantom study. Neurosurg Focus. 2019; 47(1): E19, doi: 10.3171/2019.4.FOCUS19235, indexed in Pubmed: 31261123. 